\title{
An Operant Conditioning Task to Assess the Choice between Wheel Running and Palatable Food in Mice
}

Bastien Redon ${ }^{1,2, *}$, Imane Hurel, ${ }^{1,2}$, Giovanni Marsicano ${ }^{1,2}$ and Francis Chaouloff ${ }^{1,2}$

${ }^{1}$ Inserm U1215, Neurocentre Magendie, Team Endocannabinoids \& NeuroAdaptation, Bordeaux F33077, France; ${ }^{2}$ Université de Bordeaux, Bordeaux-F33077, France

*For correspondence: bastien.redon@inserm.fr

\begin{abstract}
[Abstract] Wheel running, especially in the homecage, has been widely used to study the neurobiology of exercise because animal tends to use it voluntarily. However, as for each reward, its consumption (in the present case, running performance) does not specifically provide information on its incentive value, i.e., the extent to which animals are motivated to run independently from their consumption of that reward. This is a major drawback, especially when focusing on the neurobiology governing the pathological imbalances between exercise and e.g., feeding (obesity, anorexia nervosa). Yet, few studies have shown that operant conditioning wherein wheel-running is used as a reinforcer that can be "consumed" after nose-poking or lever-pressing allows to distinguish motivation from consumption. Thus, nose-poking or lever-pressing under a progressive ratio schedule of reinforcement in animals trained under fixed ratio reinforcement schedules provides, through the so-called breakpoint, an index of running motivation. As compared to wheel-running, numerous studies have used food as a reinforcer, which helped to uncover the neurobiology of feeding. However, to our knowledge, there is no paradigm allowing the assessment of the choice between running and feeding when presented in concurrence, with the possibility to measure a priori the motivation for each reward. Herein, we describe a protocol that first permits to measure the drive for each of these two rewards before it allows to measure the preference for one over the other in a reward choice setting. This paradigm could help to better characterize the neurobiology underlying pathological imbalances between physical activity and feeding, which is the core feature of eating disorders.
\end{abstract}

Keywords: Operant conditioning, Choice, Reward, Motivation, Wheel-running, Palatable food, Exercise, Physical Activity

[Background] Physical inactivity is a growing burden for society nowadays, and it finds its root in a lack of motivation to engage in or to adhere to a long-term exercise program (Ekkekakis et al., 2008). More broadly, eating disorders result from an alteration of the energy balance, between caloric intake (e.g., food intake) and expenses (e.g., physical activity). Interestingly, such an alteration finds its origin at the motivation level. It is thus of prime importance to study in a combined manner the motivation for physical activity with the motivation for food intake. To unravel the neurobiology behind exercise motivation, most studies have used the running wheel paradigm as wheel-running is a volitional and well-conserved behavior with highly rewarding properties (Sherwin, 1998). Indeed wheel running is able to reinforce operant conditioning (Belke, 1997), even when the duration of running is minimal (Iversen, 1993). The 
basic principle of operant conditioning is to render the reward access contingent to the realization of an effort (e.g., lever pressing, nose-poking). By progressively increasing the effort, it allows estimating the animal's motivation for this reward. However, studying motivation for physical activity in the context of eating disorders requires to compare it with the motivation for food intake in order to integrate both aspects of energy balance. Noteworthy is the observation that the comparison between the maximal efforts the animal displays for each reward taken individually can be misleading because it doesn't represent the preference for one reward over another in the natural context of a choice between these rewards (Cantin et al., 2010, in this paper the authors designed an operant conditioning protocol to assess the choice between cocaine and sucrose). A previous study investigated the choice between wheel-running and sucrose in a T-maze (Correa et al., 2016). Although providing a cue with regard to the preference between the rewards under effort-less conditions, this approach does not allow to dissect the motivation for each reward from its mere consumption. To our knowledge, the protocol we are describing here is the first to allow the a priori assessment of motivation for both wheel running and palatable food, and the preference for one over the other in a concurrent choice context using operant conditioning (Muguruza et al., 2019). Such a protocol allows access to both sides of the energy balance as to study the mechanisms involved in such a regulation. Furthermore, by modulating the effort that is required for each reward, one can externally affect the preference for one reward over the other one, hence helping to assess the neurobiological grounds governing each preference level. As it is the case for most studies investigating reward neurobiology, our protocol bears some limitations. Even though animals are always exposed to the operant chambers during their active phase (dark phase), this daily single and restricted exposure cannot fully recapitulate the human situation. Indeed, the animals are exposed to this concurrent choice once a day in a different environment than their homecage when humans have permanently to choose between concurrent rewarding activities. One possibility to circumvent this limitation would be to host the animal in an operant chamber where it would access running and feeding activities, these being fully contingent to an effort (namely nose poking).

\section{Materials and Reagents}

1. C57BI/6N mouse line (Janvier Labs): used to validate the protocol (7-8 weeks old) Notes:

a. The use of other mouse lines is possible in the following protocol given that no learning or motor deficits are observed in this line. Hereafter we show example of graphs based on the use of a transgenic line, the SimCB1 mouse line (C57BI/6N background).

b. The size and the strength of the mouse are important to take into account so as to screw the wheel properly, avoiding a spontaneous rotation (too loose) or the impossibility for the animal to make it turn (too tight).

2. Safe A03 (Safe Diet, U8200G10R, stored at room temperature after opening) contains 3.4 $\mathrm{Kcal} / \mathrm{g}$ (61.3 percent from carbohydrates, 25.2 percent from proteins and 13.5 percent from fat): Standard homecage food 
3. Dustless Precision Pellets ${ }^{\circledR}, 20 \mathrm{mg}$, Rodent Purified Diet, Chocolate Flavor (BioServ, Product \#F05301, stored at room temperature with silica gel after opening) contains $3.60 \mathrm{Kcal} / \mathrm{g}(65.6$ percent from carbohydrates, 20.6 percent from proteins and 13.9 percent from fat) with an addition of a chocolate flavoring : Palatable food reward

4. Phagospray ${ }^{\circledR}$ (Laboratoire Phagogène, Christeyns France). Antimicrobial disinfectant spray designed for non-invasive medical devices and surfaces: used to clean the operant chambers between animals

\section{Equipment}

1. Apparatus: Operant conditioning chamber (Imetronic, 156 avenue Jean Jaurès 33600 Pessac, France, customized apparatus) (Figure 1)

Individual operant conditioning chamber ( $28 \mathrm{~cm}$ long $\times 26 \mathrm{~cm}$ wide $\times 38 \mathrm{~cm}$ high) placed in a wooden case $(60 \mathrm{~cm}$ long $\times 62 \mathrm{~cm}$ wide $\times 49 \mathrm{~cm}$ high) ventilated to ensure air circulation and background noise. The rear side has a $20 \mathrm{~cm}$ diameter wheel mounted at the center, surrounded by two nose poke ports, with a brake pad on top of it to be able to lock or unlock it. The left panel has a food tray placed at the center surrounded by two nose poke ports. Both panels (rear or left) can be hidden using grey Perspex depending on the task. The floor consists of a grid above a drawer filled with litter (same litter as animals' homecages). All operant chambers are connected to a computer through an interface allowing (i) the allocation of files and exercise (see following sections) for each animal/cage and (ii) real-time visualization of animal behavior and recordings. For a given batch of animal, the active nose poke port is allocated to be the right one for half of the animals, and the left for the other half in order to counterbalance the position.

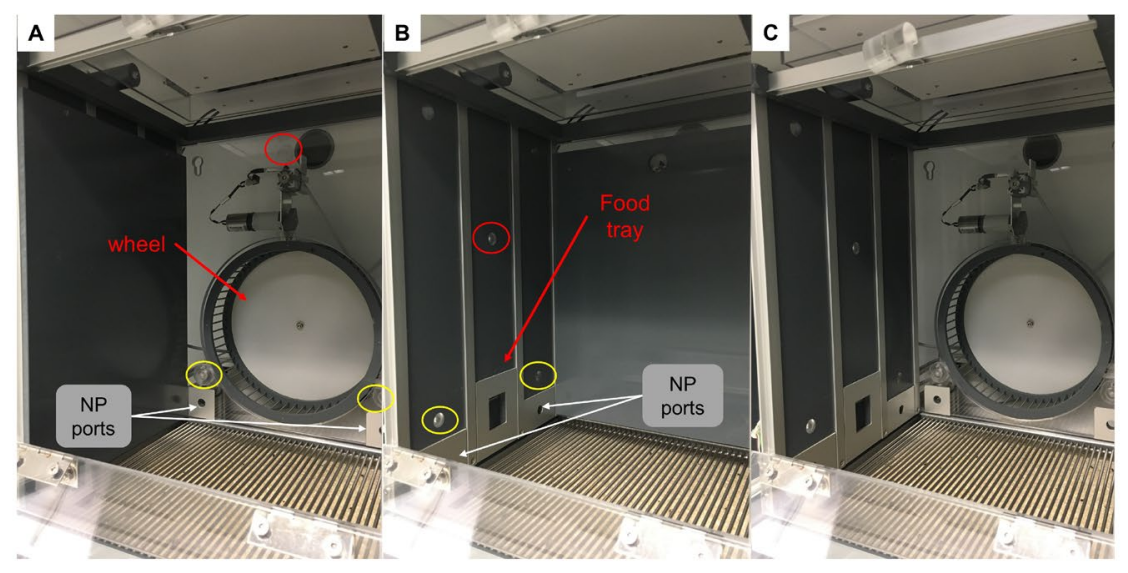

Figure 1. Operant chambers for wheel running and palatable food. A. The configuration for wheel running involves a wheel mounted on the rear side surrounded by two nose poke ports associated with light cues (yellow circles) that can be paired with another light cue (red circle) on the top of the wheel (the left panel being covered by gray Perspex). The floor is constituted of a metal grid on the top of a litter drawer. B. The configuration for palatable food involves a 
food tray on the left panel surrounded by two nose poke ports associated with light cues (yellow circle) that can be paired with another light cue (red circle) on the top of the food tray (the rear side covered by gray Perspex). The floor is made of a metal grid on the top of a litter drawer. C. The choice configuration involves the removal of the two gray Perspex sides to free the access to both rewards. The floor is made of a metal grid on the top of a litter drawer. Illustration taken from Hurel et al. (2019).

\section{Software}

1. Polywheel 5.2 .2 (16/04/2015) Imetronic, 156 avenue Jean Jaurès 33600 Pessac, France: Software used on-line allowing the allocation of exercise (see section "procedure" and subsections "exercise design"), and the real-time acquisition of data from the operant chambers

2. Poly files V4.5.2 (09/04/2018) Imetronic, 156 avenue Jean Jaurès 33600 Pessac, France: Software used off-line to extract data using pre-defined variables into Excel files

3. GraphPad Prism version 8 (GraphPad Software 2365 Northside Dr., suite 560, San Diego, CA 92108): Data analysis

\section{Procedure}

Note: All experiments are performed during the dark phase of the light/dark cycle to be in the active phase. We are thus working in a partly inverted $12 \mathrm{~h} / 12 \mathrm{~h}$ cycle with lights off at $10 \mathrm{AM}$ and on at 10 PM.

\section{A. Acclimation}

1. Habituate the animal to the inverted cycle one week before starting the protocol.

2. Individualize the animals.

3. Three days before starting the protocol, give 3-4 chocolate food pellets in the homecage to avoid neophobia.

B. General description of operant conditioning sessions

1. Select the specific file for each mouse (one per reinforcement schedule and per mouse e.g., FR1_mouse1).

2. Select the corresponding exercise (cf. Procedure: Exercises design).

3. Place the mouse inside the operant chamber.

4. Start the recording.

5. At the end of the session, remove the mouse from the operant chamber.

If the session is for palatable food:

6. Count the remaining pellets in 1) the food tray and 2) the litter below the grid. 
For both wheel running and palatable food:

7. Clean the operant chamber using Phagospray ${ }^{\circledR}$ and, if within a palatable food session, clean the food tray with dry air spray.

For habituation, FR1 and FR3 sessions:

8. Go back to Step B1 for the second session of the day (second reward).

Note: To avoid the effect of one reward over the other on the same day, half of the animal must start with a session of wheel running, the other half with a session of palatable food, the order being inverted each day.

C. Habituation (2 days)

1. Exercise design

\section{Wheel-running}

a. The sessions last 30 min each.

b. The cue light paired with the wheel (on top of it) turns on at the beginning of the session and remains on for $30 \mathrm{~min}$.

Note: The wheel-paired cue light indicates the availability of the wheel. Thus, it remains on all over the habituation session.

c. The wheel remains unlocked all session-long.

\section{Palatable food}

a. The sessions last 30 min each.

b. The cue light paired with the food tray (on top of it) turns on at the beginning of the session and remains on for $30 \mathrm{~min}$.

Note: The food tray-paired cue light indicates the delivery of food pellets in the food tray. Thus, it remains on all over the habituation session.

c. Seventeen pellets are distributed consecutively at the beginning of the session.

2. Practical steps

a. Cover all nose poke ports with the appropriate metal piece.

b. Place the appropriate Perspex wall to cover either the food tray or the wheel and their corresponding nose poke ports.

c. Perform two sessions (1/day) for both rewards applying the instructions of Procedure B.

d. No criteria are needed to be met to move on to the next step.

D. Fixed ratio 1 schedule of reinforcement (5 days)

1. Exercise design

\section{Wheel-running (Figure 2)}

a. The sessions last $30 \mathrm{~min}$.

b. The sessions start without any light cue, the wheel being blocked by the brake pad. 
c. One nose poke (NP) in the active port triggers a rewarded sequence with simultaneous:

i. Five-second NP-paired cue light turns on.

Note: This NP-paired cue light is a conditioned stimulus indicating that the effort required to get access to the wheel is completed.

ii. Twenty-second wheel-paired cue light turns on.

Note: The wheel-paired cue light is a conditioned stimulus indicating the availability of the wheel.

iii. Twenty-second unlocking of the wheel (removing the brake pad).

d. Nose-poking during the rewarded sequence is recorded but has no functional consequence.

e. After the rewarded sequence, the program goes back to the first step until the next NP.

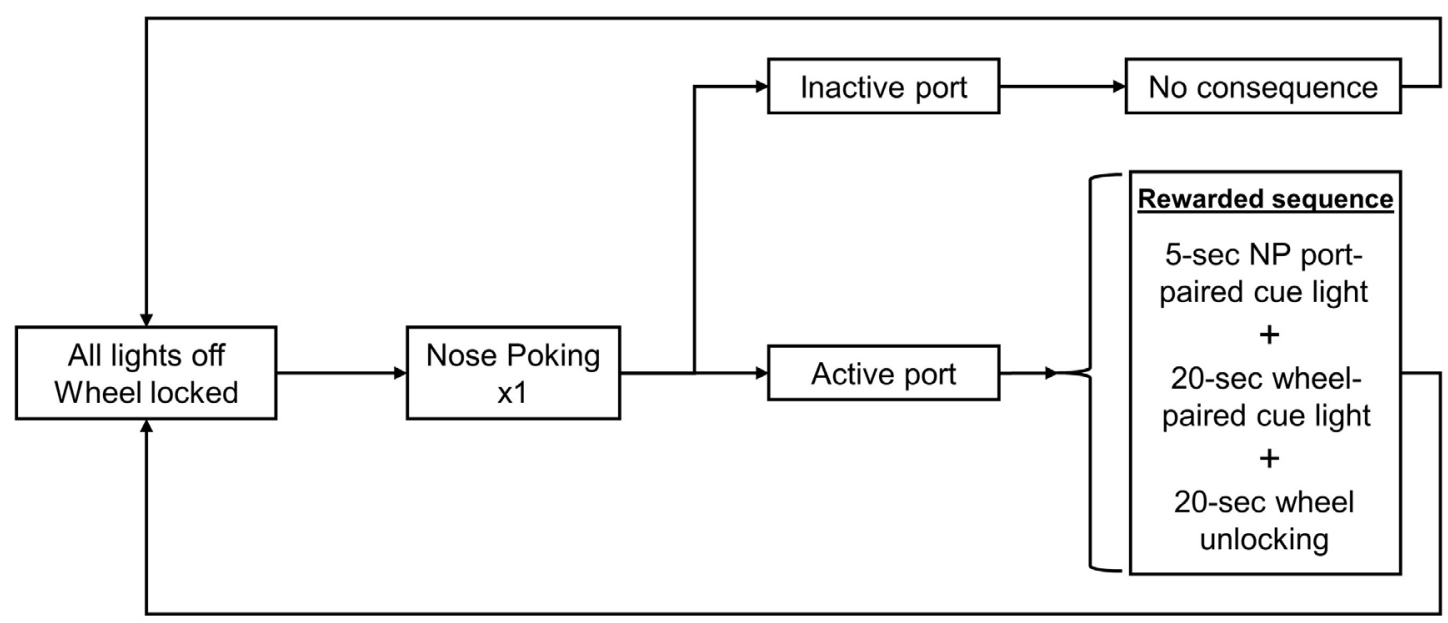

Figure 2. Logigram of Fixed ratio 1 schedule of reinforcement for wheel running. During the 30-min session, one nose poke in the active port gives access to a rewarded sequence of $20 \mathrm{~s}$ before going back to the initial condition. NP: Nose Poke.

\section{Palatable food (Figure 3)}

a. The sessions last $30 \mathrm{~min}$.

b. The sessions start without any light cue, the food tray being available but empty.

c. One NP in the active port triggers a rewarded sequence with simultaneous:

i. Five-second NP-paired cue light turns on.

Note: This NP-paired cue light is a conditioned stimulus indicating that the effort required to get one food pellet is completed.

ii. Fifteen-second food tray-paired cue light turns on.

Note: The food tray-paired cue light is a conditioned stimulus indicating the delivery of one food pellet.

iii. One chocolate food pellet is distributed.

d. Nose-poking during the rewarded sequence is recorded but has no functional consequence.

e. After the rewarded sequence, the program goes back to the first step until the next NP. 


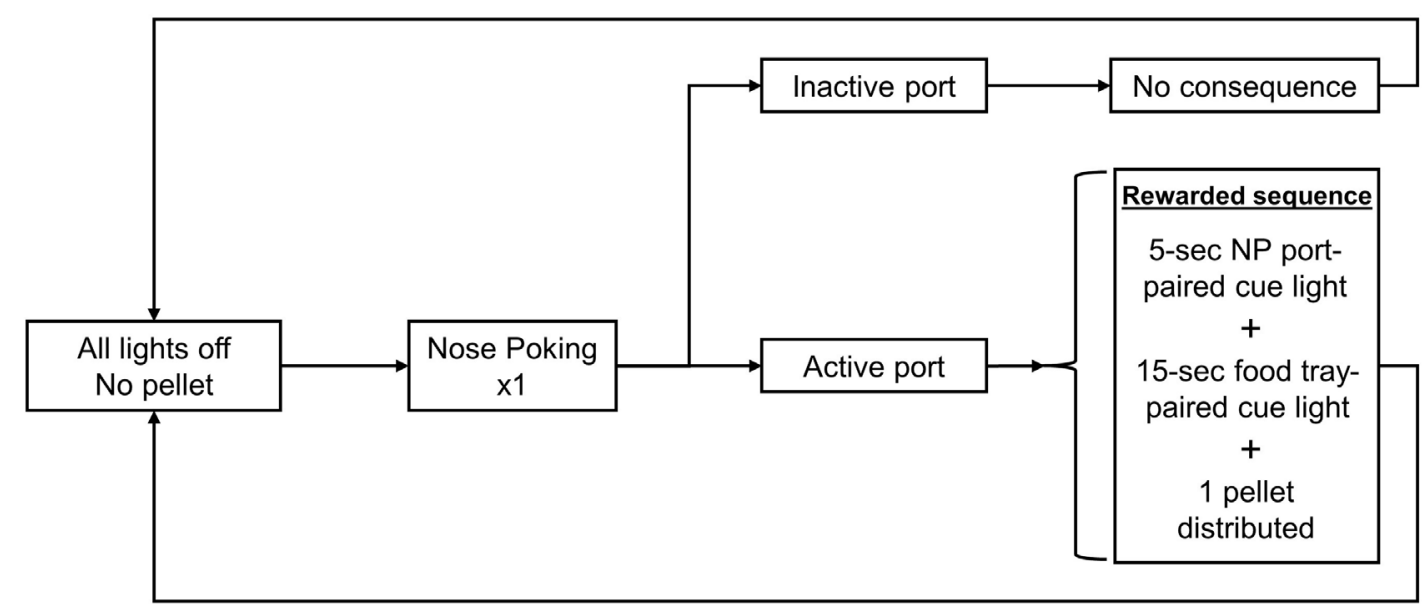

Figure 3. Logigram of Fixed ratio 1 schedule of reinforcement for palatable food. During the 30-min session, one nose poke in the active port gives access to a rewarded sequence of $15 \mathrm{~s}$ before going back to the initial condition. NP: Nose Poke.

2. Practical steps

Note: In order to facilitate the learning, the animals are mildly food-restricted for the 2-3 first sessions so as to reach a stable $10 \%$ decrease in body weight.

a. Remove all the metal pieces from nose poke ports so as to render them accessible.

b. Place the appropriate Perspex wall to cover either the food tray or the wheel and their corresponding nose poke ports.

c. Perform five sessions (1/day) for both rewards following the instructions of Procedure B.

d. No criteria are needed to be met to move on to the next step.

E. Fixed ratio 3 schedule of reinforcement (5 days)

1. Exercise design

\section{Wheel-running (Figure 4)}

a. The sessions last $30 \mathrm{~min}$.

b. The sessions start without any light cue, the wheel being blocked by the brake pad.

c. Three consecutive NPs in the active port trigger a rewarded sequence with simultaneous:

i. Five-second NP-paired cue light turns on.

ii. Twenty-second wheel-paired cue light turns on.

iii. Twenty-second unlocking of the wheel (removing the brake pad).

d. Nose-poking during the rewarded sequence is recorded but has no functional consequence.

e. After the rewarded sequence, the program goes back to the first step until the next NP. 


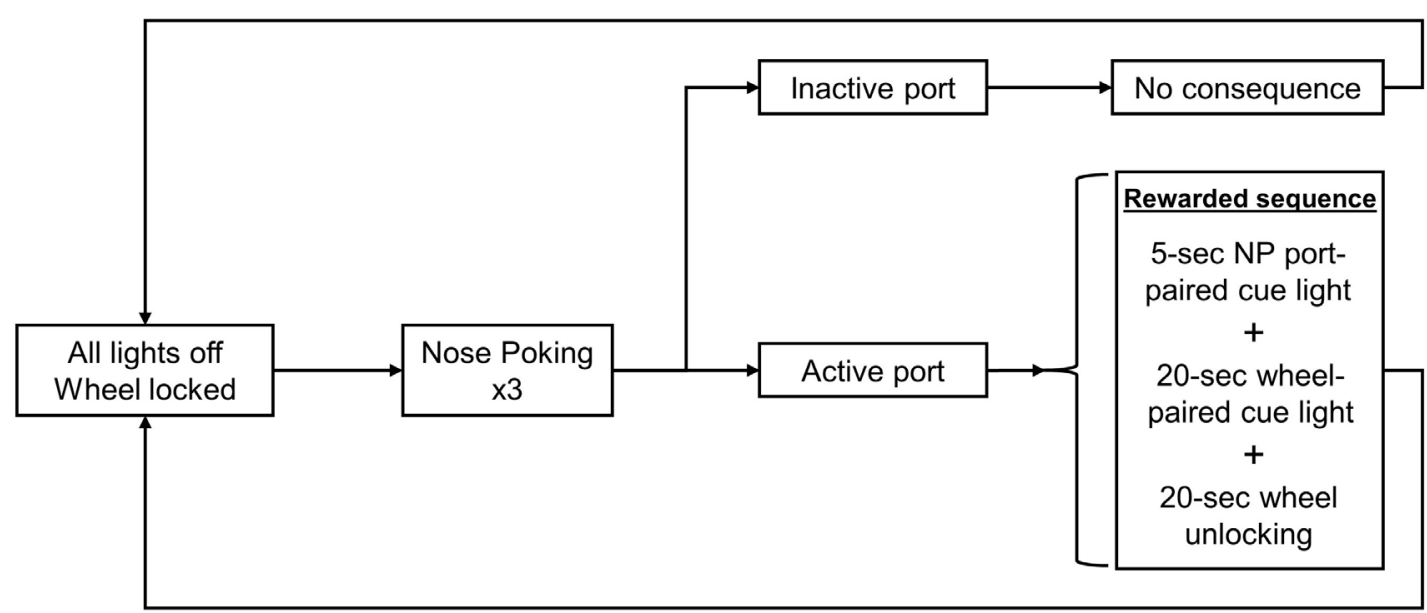

Figure 4. Logigram of Fixed ratio 3 schedule of reinforcement for wheel running. During the 30-min session, three consecutive nose pokes in the active port give access to a rewarded sequence of $20 \mathrm{~s}$ before going back to the initial condition. NP: Nose Poke.

\section{Palatable food (Figure 5)}

a. The sessions last $30 \mathrm{~min}$.

b. The sessions start without any light cue, the food tray being available but empty.

c. Three consecutive NPs in the active port trigger a rewarded sequence with simultaneous:

i. Five-second NP-paired cue light turns on.

ii. Fifteen-second food tray-paired cue light turns on.

iii. One chocolate food pellet is distributed.

d. Nose-poking during the rewarded sequence is recorded but has no functional consequence.

e. After the rewarded sequence, the program goes back to the first step until the next NP.

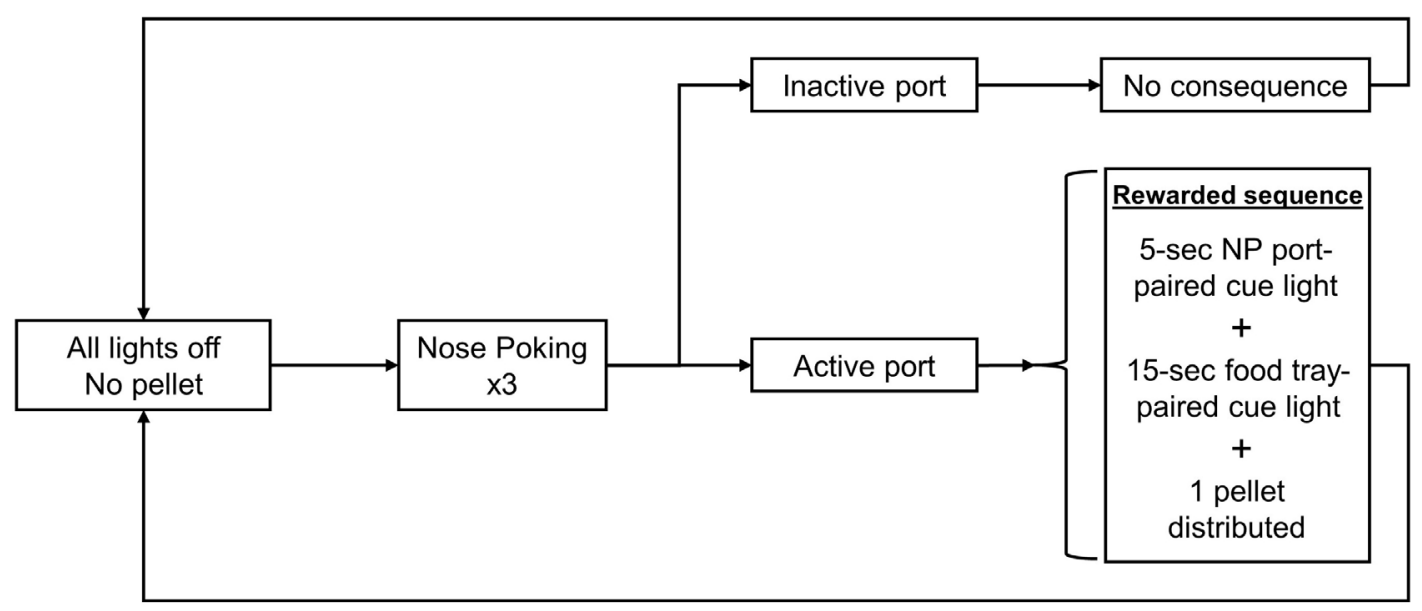

Figure 5. Logigram of Fixed ratio 3 schedule of reinforcement for palatable food. During the 30-min session, three consecutive nose pokes in the active port give access to a rewarded sequence of $15 \mathrm{~s}$ before going back to the initial condition. NP: Nose Poke. 
2. Practical steps

a. Remove all the metal pieces from nose poke ports so as to render them accessible.

b. Place the appropriate Perspex wall to cover either the food tray or the wheel and their corresponding nose poke ports.

c. Perform five sessions (1/day) for both rewards following the instructions of Procedure $B$.

d. All animals need to meet the following criteria:

i. Stability of performance over 3 consecutive days $(<20 \%$ variation in the total number of active nose pokes).

ii. Discrimination index over $75 \%$ (see "Data analysis" part).

F. Progressive ratio schedule of reinforcement (2 days)

Note:

1. A 30 min session for the assessment of wheel running and palatable food breakpoints was chosen in order to have a comparable duration for both rewards without reaching satiety in the palatable food session.

2. This duration can be adapted by investigators depending on the information needed from the progressive ratio assessment.

1. Exercise design

\section{Wheel-running (Figure 6)}

a. The session lasts $30 \mathrm{~min}$.

b. The session starts without any light cue, the wheel being blocked by the brake pad.

c. Performing $n \times 3 \mathrm{NP}$ (with " $\mathrm{n}$ " being the rank number of the reward accessed e.g., the first access to the wheel required $1 \times 3=3 \mathrm{NP}$, the second one $2 \times 3=6 \mathrm{NP}$ etc. The sequence is thus as follows: $3,6,9,12,15,18,21,24,27 \ldots)$ in the active port trigger a rewarded sequence with simultaneous:

i. Five-second NP-paired cue light turns on.

ii. Twenty-second wheel-paired cue light turns on.

iii. Twenty-second unlocking of the wheel (removing the brake pad).

d. Nose-poking during the rewarded sequence is recorded but has no functional consequence.

e. After the rewarded sequence, the program goes back to the first step until the next NP.

f. The last ratio completed ( $\mathrm{n}_{\text {final }} \times 3 \mathrm{NP}$ ) is called the breakpoint: it is the maximum number of nose pokes the animal accepts to perform to obtain the reward in a given session. 


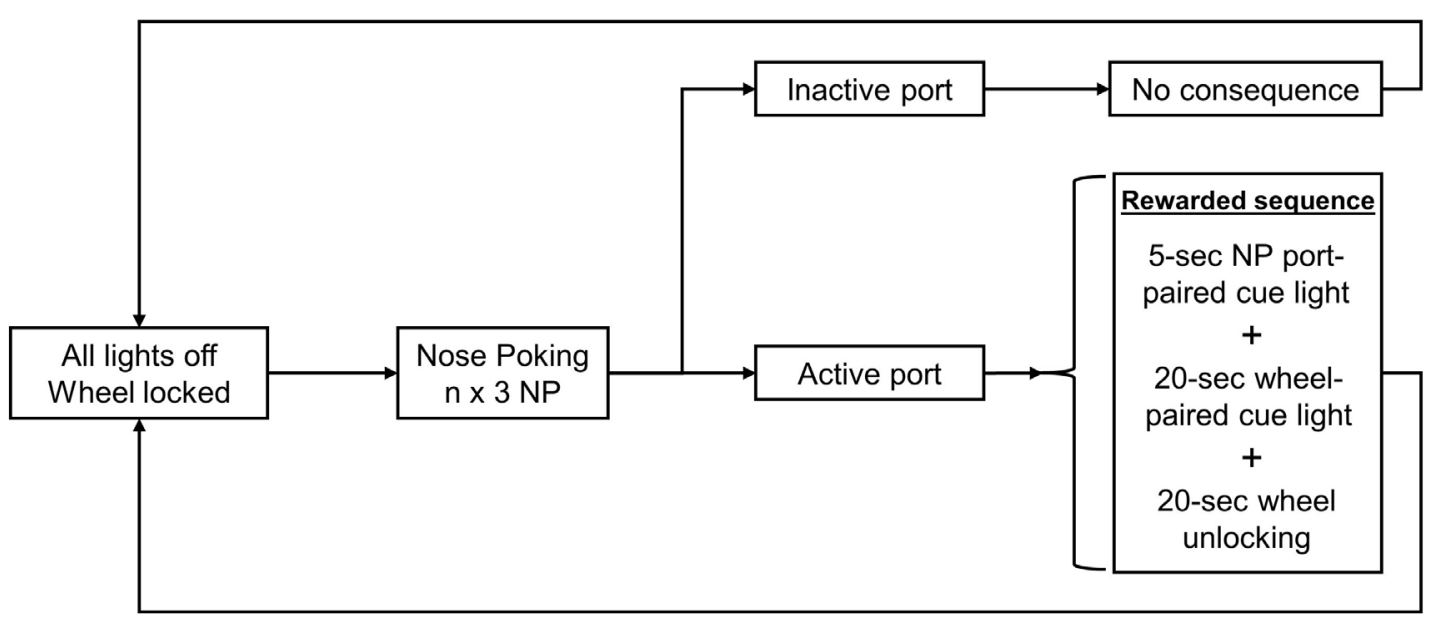

Figure 6. Logigram of Progressive ratio schedule of reinforcement for wheel running.

During the 30 -min session, the effort required to unlock the wheel is progressively increased between each rewarded sequence. The last ratio completed by the animal is the breakpoint. NP: nose poke; $n$ : rank number of reward accessed.

\section{Palatable food (Figure 7)}

a. The session lasts $30 \mathrm{~min}$.

b. The session starts without any light cue, the food tray being available but empty.

c. Performing $\mathrm{n} \times 3 \mathrm{NP}$ (with " $\mathrm{n}$ " being the rank number of the reward accessed e.g., the first access to the wheel required $1 \times 3=3 \mathrm{NP}$, the second one $2 \times 3=6 \mathrm{NP}$ etc. The sequence is thus as follows: $3,6,9,12,15,18,21,24,27 \ldots)$ in the active port trigger a rewarded sequence with simultaneous:

i. Five-second NP-paired cue light turns on.

ii. Fifteen-second food tray-paired cue light turns on.

iii. One chocolate food pellet is distributed.

d. Nose-poking during the rewarded sequence is recorded but has no functional consequence.

e. After the rewarded sequence, the program goes back to the first step until the next NP. 


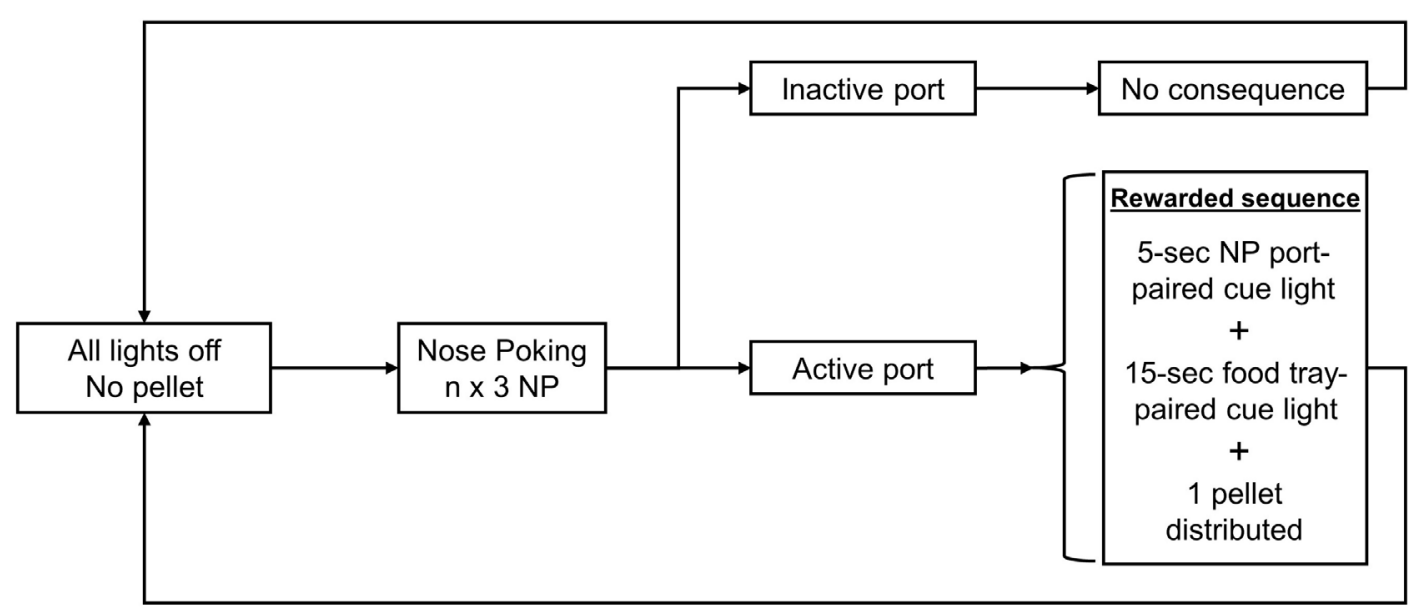

Figure 7. Logigram of progressive ratio schedule of reinforcement for palatable food. During the 30 -min session, the effort required to obtain a pellet is progressively increased between each rewarded sequence. The last ratio completed by the animal is the breakpoint. NP: nose poke; n: rank number of reward accessed.

2. Practical steps

Note: This test is intended to assess the maximum effort an animal is willing to expend to get access to the reward. Accordingly, one unique 30-min session per day is performed for each animal given the highly demanding nature of the task.

a. Remove all the metal pieces from nose poke ports so as to render them accessible.

b. Place the appropriate Perspex wall to cover either the food tray or the wheel and their corresponding nose poke ports.

c. The first day, half of the animal will undergo one unique session of PR for wheel running, the other half one unique session of PR for palatable food. For this apply the instructions of Procedure B from Step B1 to Step B7.

d. The second day, the animal undergoes one unique session of $P R$ for the reward not given on the first day. For this apply the instructions of Procedure B from Step B1 to Step B7.

e. No criteria are needed to be met to move on to the next step.

G. Fixed Ratio 3 Schedule of reinforcement post-progressive ratio (1 day)

Note: This step is intended to ensure that the performances of the animals go back to pre-PR levels.

1. Exercise design

Follow instructions of Procedure E1

2. Practical steps

Follow instructions of Procedure E2 for 1 day 


\section{H. Choice (Figure 8) (Video 1)}

1. Exercise design

a. The sessions last $60 \mathrm{~min}$.

b. The sessions start without any light cue, the wheel being blocked by the brake pad, and the food tray being available but empty.

c. If the animal performs 3 consecutive NP in the active port for the wheel running, it gets access to a wheel-rewarded sequence:

i. Simultaneous:

Five-second NP-paired cue turns on

Twenty-second wheel-paired cue turns on

Twenty-second wheel unlocking

ii. Nose-poking during the rewarded sequence is recorded but has no functional consequence.

iii. At the end of the rewarded sequence, a green ceiling light turns on for 5 additional seconds during which no reward is accessible. Nose poking is recorded but has no functional consequence.

iv. Then, the program goes back to the start of Step H1b until the next NP.

d. If the animal performs 3 consecutive NP in the active port for the palatable food, it gets access to a palatable food-rewarded sequence:

i. Simultaneous:

Five-second NP-paired cue turns on

Fifteen-second food tray-paired cue turns on

One chocolate food pellet is distributed

ii. Nose-poking during the rewarded sequence is recorded but has no functional consequence.

iii. At the end of the rewarded sequence, a green ceiling light turns on for 5 additional seconds during which no reward is accessible. Nose poking is recorded but has no functional consequence.

iv. Then, the program goes back to the start of step H1b until the next NP. 


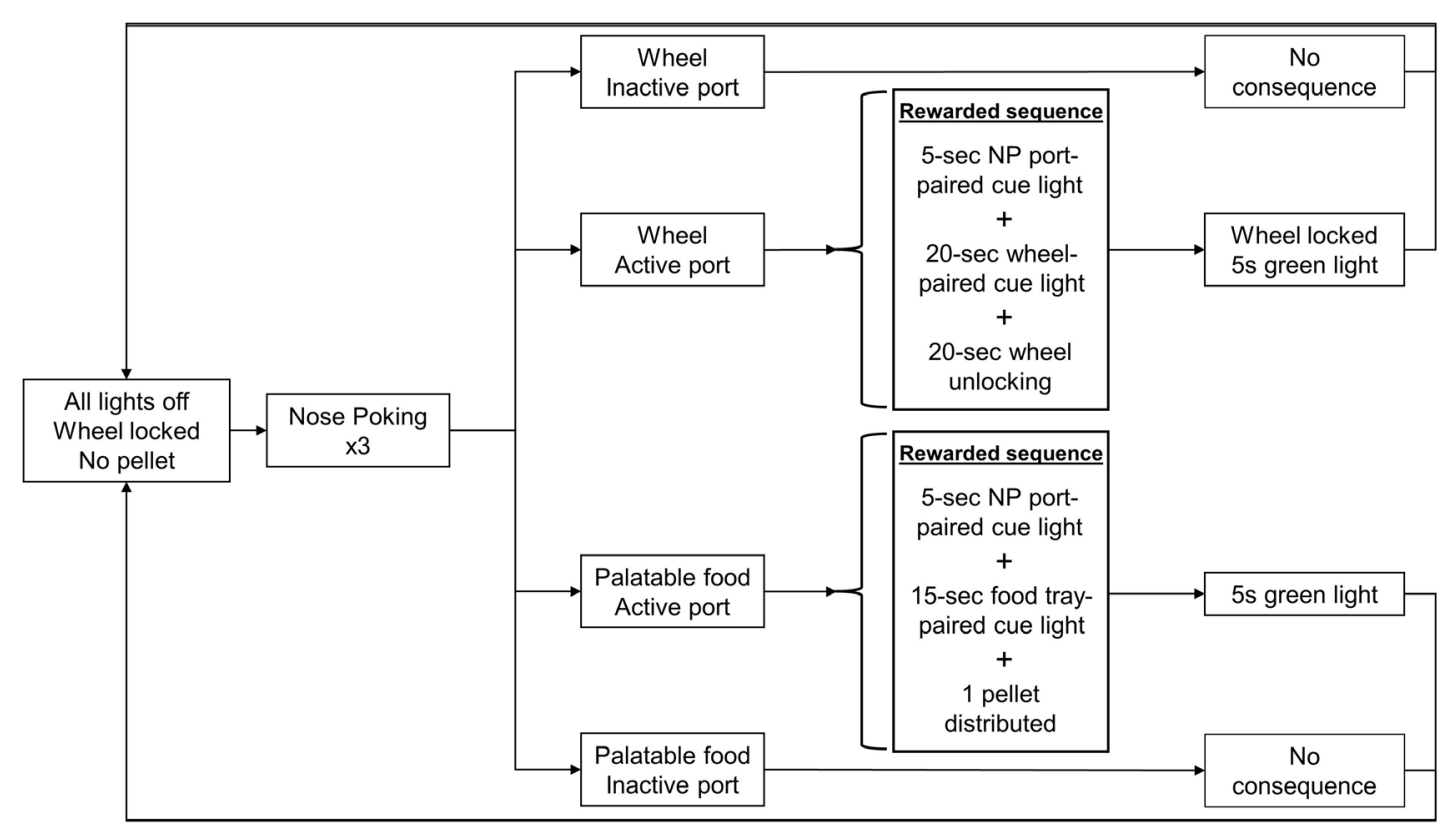

Figure 8. Logigram of the choice between wheel running and palatable food. Nose poking gives access to one reward but the choice is mutually exclusive, meaning that choosing one reward excludes the possibility to obtain the other reward for a given time.

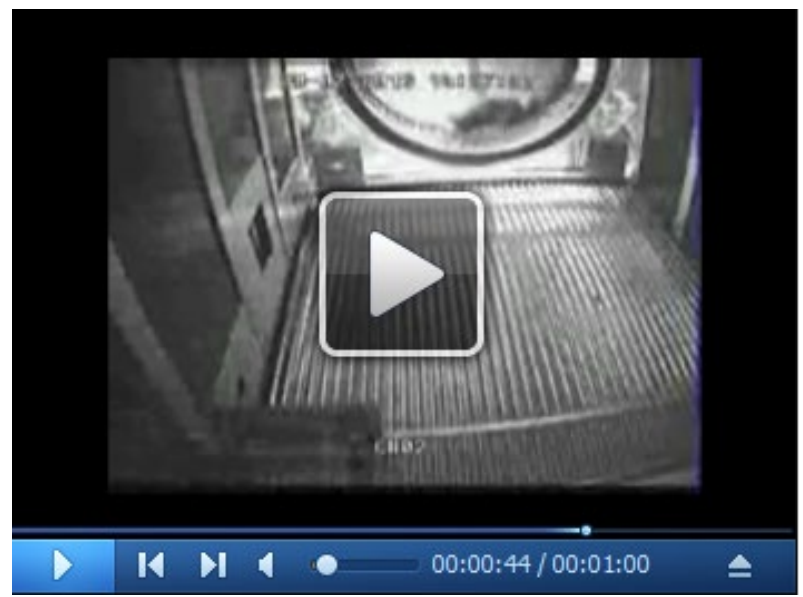

Video 1. Choice session showing (i) a food-rewarded sequence followed by (ii) a wheelrewarded sequence. (All experiments obeyed the French (Décret 2013-118) and European (2010/63/EU) rules on animal experimentation with authorization numbers 33-063-69 (F.C.) and A33-063-098 (Animal facilities).)

2. Practical steps

a. Choice in fed animals (5 days)

i. Remove all the metal pieces from nose poke ports so as to render them accessible.

ii. Remove all the Perspex walls so as to render both rewards accessible.

iii. Perform five sessions applying the instructions of Procedure B from Step B1 to Step B7.

b. Choice under mild food restriction 
i. In the hour preceding the dark phase before the first session, remove the food from the home cage.

ii. In the hour preceding the dark phase, weigh the animals.

iii. Remove all the metal pieces from nose poke ports so as to render them accessible.

iv. Remove all the Perspex walls so as to render both rewards accessible.

v. Perform one session applying the instructions of Procedure B from Step B1 to Step B7.

vi. Calculate the amount of food to give:

1) Seventy percent of mean daily consumption.

2) Subtract the amount eaten during the session.

vii. Give the food in the home cage at least $1 \mathrm{~h}$ after the end of the operant conditioning session

viii. Perform the other sessions on the following days applying Steps $\mathrm{H} 2 \mathrm{~b}$ ii to H2b vii.

Note: The body weight must be comprised between 85 and $90 \%$ of the normal body weight. If it is above or below this range, reduce or increase the amount of food accordingly.

\section{Data analysis}

A. Inclusion criteria

1. Learning of the contingency between nose-poking and reward access is assessed by the discrimination index $=\frac{\text { Number of active nose pokes }}{\text { Total number of nose pokes }}$

2. Animals should display a stable performance over 3 consecutive sessions at the end of the FR3 step: $<20 \%$ variation of the number of active nose pokes.

3. Animals that do not meet the preceding criteria are discarded from the experiment.

B. All protocol

1. Number of active nose pokes: (Figure 9)

Analyzed by two-way ANOVA (repeated measures) for each step of the protocol separately (FR1, FR3 and Choice).

2. Number of inactive nose pokes (Figure 9).

3. Running duration per rewarded sequence

a. This index gives an insight into how much the animal runs every time it gains access to the wheel (for details see Muguruza et al. 2019).

b. Running duration per rewarded sequence $=\frac{\text { total running duration }(\text { seconds) }}{\text { number of rewarded sequences }}$.

c. Analyzed with two-way ANOVA (repeated measures) for each step of the protocol separately (FR1, FR3 and Choice).

4. Pellets consumed

a. This index measures the consumption of the rewards obtained (to ensure that the pellets earned are consumed). 
b. Analyzed with two-way ANOVA (repeated measures) for each step of the protocol separately (FR1, FR3 and Choice).

C. Progressive Ratio (Figure 9)

1. Number of active nose pokes: (Figure 9) Analyzed by two-tailed Student's $t$-test.

2. Breakpoint: (Figure 9) Analyzed by two-tailed Student's $t$-test.

The breakpoint is defined as the last rewarded step reached during the PR session, in other words, it corresponds to the maximum number of nose pokes the animal accepts to perform to get access to one reward in a given session.

3. Pellets consumed: Analyzed by two-tailed Student's $t$-test.

4. If the results do not follow normality, C.1, C.2 and C.3 should be analyzed using a Mann-Whitney test.
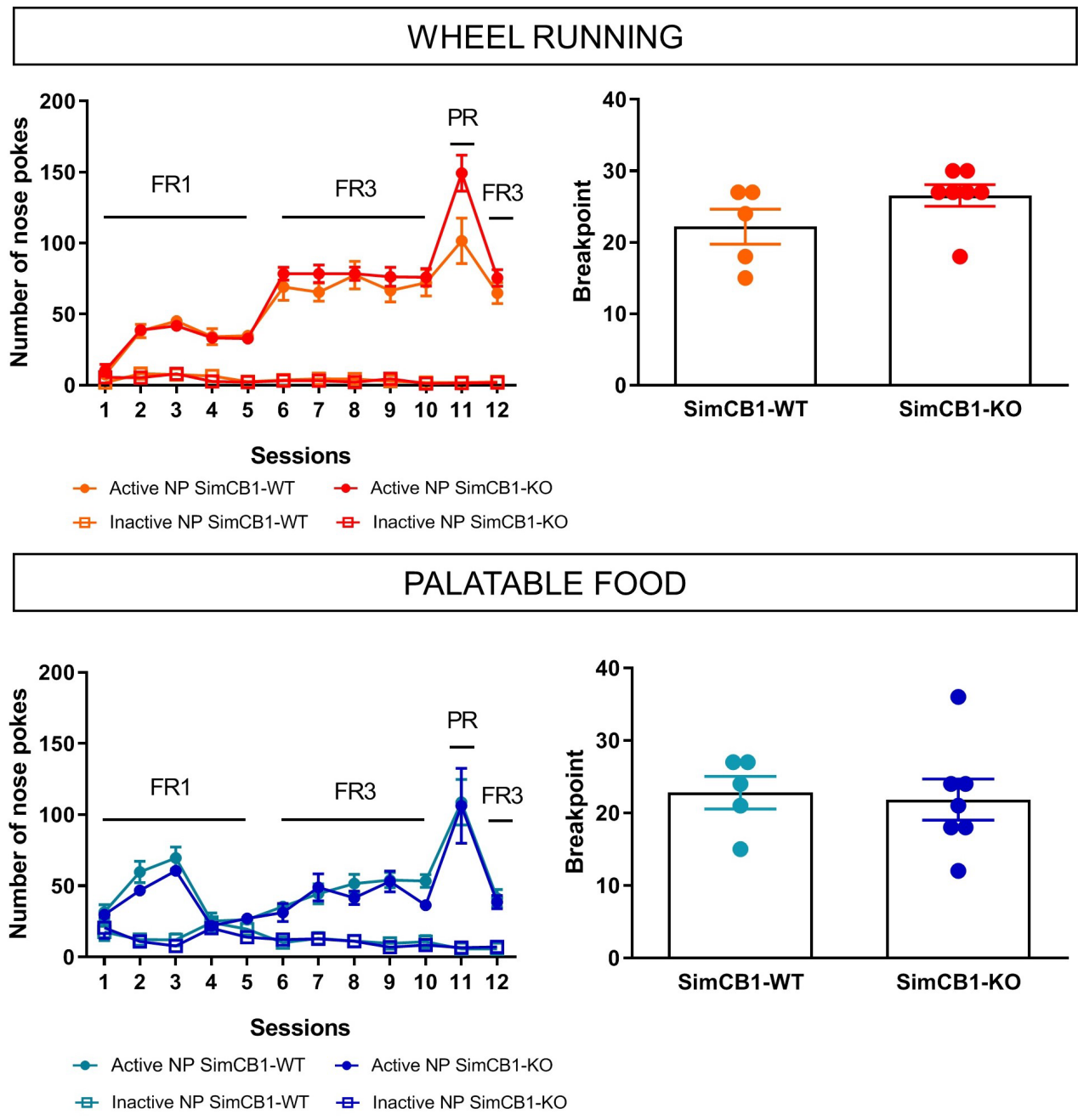

Figure 9. Representation of the number of nose pokes and breakpoints when each reward is presented separately (FR1, FR3 and PR). The graphs presented here are data obtained from the Sim-CB1 mouse line, comparing knock-out mice (Sim-CB1-KO) to their wild-type littermates (Sim-CB1-WT). This transgenic mouse line bears a deletion of the cannabinoid type- 
1 receptor (CB1R) in Sim1-positive cells, mainly found in the paraventricular nucleus of the hypothalamus (PVN). For both wheel running (upper panel) and palatable food (bottom panel), the number of nose pokes performed all over fixed- and progressive-ratio schedule of reinforcement (left) and the breakpoint reached during the progressive ratio session (right) are represented. NP: Nose Poke.

\section{Choice sessions}

1. Number of active nose pokes (Figure 10): Analyzed by two-way ANOVA (repeated measures) for each step separately (ad libitum fed and food restricted).

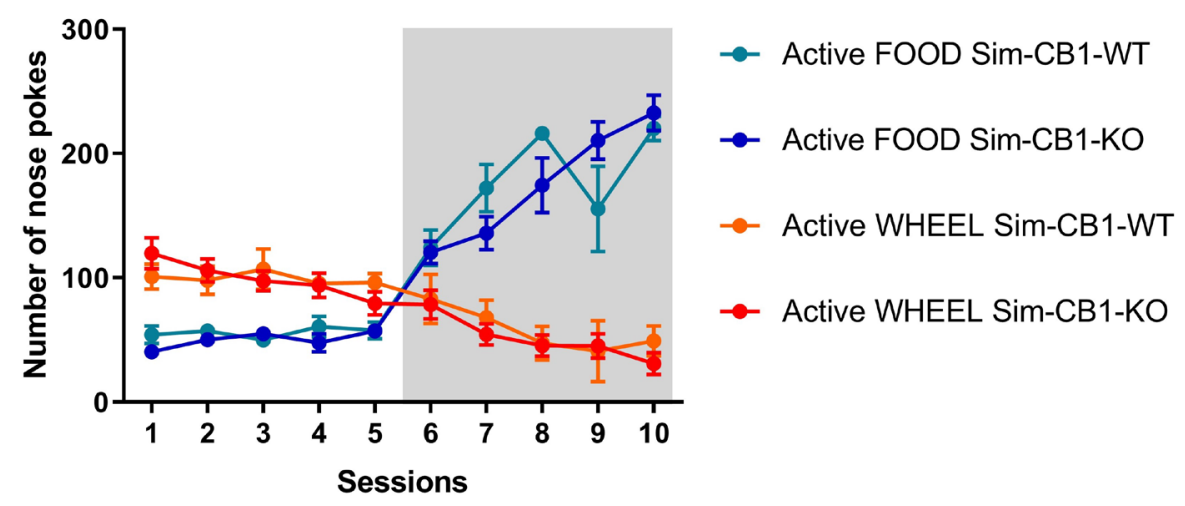

Figure 10. Representation of the number of active nose poke during the choice sessions for the Sim-CB1 mouse line (WT vs. KO). The graphs presented here are the data obtained from the Sim-CB1 mouse line, comparing knock-out mice (Sim-CB1-KO) to their wild-type littermates (SimCB1-WT). This transgenic mouse line bears a deletion of the cannabinoid type1 receptor (CB1R) in Sim1-positive cells, mainly found in the paraventricular nucleus of the hypothalamus (PVN). The blank and grey areas refer to the choice sessions under ad libitum fed and food restricted conditions, respectively.

2. Preference ratio (Figure 11): Allows evaluating the performance for one reward (for example, preference for wheel running over palatable food) with an index allowing inter-individual comparisons.

Preference ratio $=\frac{\text { Number of active nose pokes for wheel running }}{\text { Total number of nose pokes }} \times 100$.

Analyzed by two-way ANOVA (repeated measures) for each step separately (ad libitum fed and food restricted). 


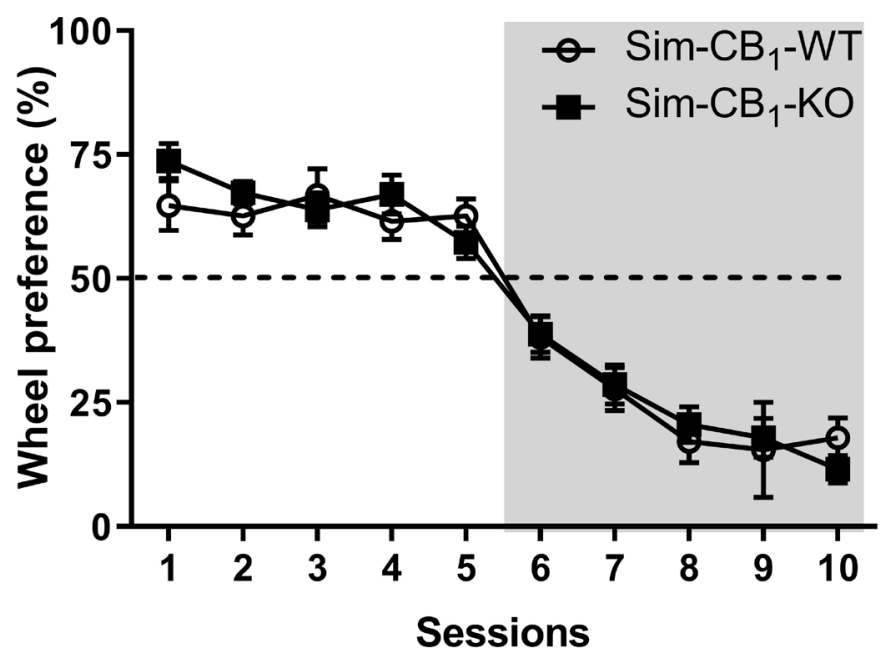

Figure 11. Preference ratio representation for the Sim-CB1 mouse line (WT vs. KO). The graphs presented here are the data obtained from the Sim-CB1 mouse line, comparing knockout mice (Sim-CB1-KO) to their wild-type littermates (Sim-CB1-WT). This transgenic mouse line bears a deletion of the cannabinoid type-1 receptor (CB1R) in Sim1-positive cells, mainly found in the paraventricular nucleus of the hypothalamus (PVN). Scores over $50 \%$ indicate a preference for wheel running and scores below $50 \%$ indicate a preference for the palatable food. White part: ad libitum fed condition choice sessions; Grey part: food restricted condition.

\section{Acknowledgments}

Our acknowledgments go to all the members of Marsicano team for their critics and useful discussions. We would like to thank particularly Dr. Serge Ahmed (IMN, CNRS UMR 5293, Bordeaux) for sharing his experience in the domain and his help in setting up the protocol. We thank Imetronic (Pessac, France) for their availability and their help to design the programs. Finally, we acknowledge the help of the members of the animal facilities and genotyping platforms in the NeuroCentre Magendie (Inserm U1215, Bordeaux, France) for their assistance.

This work was supported by Institut National de la Santé et de la Recherche Médicale (to GM), la Région Aquitaine (to GM), the University of Bordeaux (to GM), l'Agence Française de Lutte contre le Dopage (to FC), the LabEx-Bordeaux Région Aquitaine Initiative for Neuroscience (to FC), the Ministère de l'Enseignement Supérieur, de la Recherche et de l'Innovation (to BR).

\section{Competing interests}

The authors have no conflicts of interest. 


\section{Ethics}

All experiments obeyed the French (Décret 2013-118) and European (2010/63/EU) rules on animal experimentation with authorization numbers 33-063-69 (F.C.) and A33-063-098 (Animal facilities).

\section{References}

1. Belke, T. W. (1997). Running and responding reinforced by the opportunity to run: effect of reinforcer duration. J Exp Anal Behav 67(3): 337-351.

2. Cantin, L., Lenoir, M., Augier, E., Vanhille, N., Dubreucq, S., Serre, F., Vouillac, C. and Ahmed, S. H. (2010). Cocaine is low on the value ladder of rats: possible evidence for resilience to addiction. PLoS One 5(7): e11592.

3. Correa, M., Pardo, M., Bayarri, P., Lopez-Cruz, L., San Miguel, N., Valverde, O., Ledent, C. and Salamone, J. D. (2016). Choosing voluntary exercise over sucrose consumption depends upon dopamine transmission: effects of haloperidol in wild type and adenosine A(2)AKO mice. Psychopharmacology (Berl) 233(3): 393-404.

4. Ekkekakis, P., Hall, E. E. and Petruzzello, S. J. (2008). The relationship between exercise intensity and affective responses demystified: to crack the 40-year-old nut, replace the 40-yearold nutcracker! Ann Behav Med 35(2): 136-149.

5. Hurel, I., Redon, B., Scocard, A., Malezieux, M., Marsicano, G. and Chaouloff, F. (2019). Beyond the activity-based anorexia model: reinforcing values of exercise and feeding examined in stressed adolescent male and female mice. Front Pharmacol 10: 587.

6. Iversen, I. H. (1993). Techniques for establishing schedules with wheel running as reinforcement in rats. J Exp Anal Behav 60(1): 219-238.

7. Muguruza, C., Redon, B., Fois, G. R., Hurel, I., Scocard, A., Nguyen, C., Stevens, C., SoriaGomez, E., Varilh, M., Cannich, A., Daniault, J., Busquets-Garcia, A., Pelliccia, T., Caille, S., Georges, F., Marsicano, G. and Chaouloff, F. (2019). The motivation for exercise over palatable food is dictated by cannabinoid type-1 receptors. JCI Insight 4(5). pii: 126190.

8. Sherwin, C. M. (1998). Voluntary wheel running: a review and novel interpretation. Anim Behav 56(1): 11-27. 